\title{
Brain-Heart Interactions are More Diverse than Anticipated
}

\author{
Josef Finsterer ${ }^{1}$, Ana C Fiorini ${ }^{2}$, Carla A Scorza ${ }^{3}$, Fulvio A Scorza ${ }^{4}$ \\ Keywords: Arrhythmias, Autonomic nervous system, Brain-heart interactions, Cardiac, Central nervous system, Heart failure, Myocardium. \\ Indian Journal of Critical Care Medicine (2020): 10.5005/jp-journals-10071-23632
}

With interest, we read the interesting review article by Prassad Hrishi et al. about brain-heart interactions (BHIs) and the effect on the heart in general. ${ }^{1}$ The review is important since BHIs are still poorly acknowledged. However, the article has a number of shortcomings.

Generally, BHls do not only result in ventricular arrhythmias and Takotsubo cardiomyopathy (TTC) but also in a number of other direct or indirect disturbances of heart function. For example, heart function can be primarily affected by cerebral hormones or secondarily affected by involvement of the pressure arterioles or the volume load. ${ }^{2}$ It should also be discussed that drugs given for a cerebral pathology may have a direct or indirect effect on cardiac functions.

The authors do not discuss the phenomenon of sudden unexpected death in epilepsy (SUDEP) extensively. ${ }^{3}$ The cause of SUDEP is largely unknown, but a number of speculations have been raised during previous years to explain the phenomenon. ${ }^{3}$ One of these speculations is that generalized seizures trigger ventricular arrhythmias and that the patient dies from Torsades des pointes, ventricular fibrillation, or sinus arrest. Another speculation suggests that the epilepsy patient experiences TTC due to seizures and dies from intractable global-type TTC. A third speculation is that it is not a direct brain-heart problem but rather an intoxication inducing ventricular arrhythmias. This speculation relies on the fact that SUDEP predominantly occurs in patients with poorly controlled seizures and that these patients usually take more anti-seizure drugs (ASDs) than epilepsy patients with well-controlled epilepsy. Other mechanisms discussed are postictal apnea or laryngospasms and consecutive cerebral hypoxia. ${ }^{4}$

Another phenomenon that is not addressed in the review is sudden unexpected death in Parkinson's disease (SUDPAR). As in SUDEP, the pathomechanism of SUDPAR is also unknown, but there are speculations that disturbances of the autonomic nervous system, frequently affected in Parkinsonism, or the anti-Parkinson medication may play a causative role. ${ }^{5}$

Another shortcoming is the missing discussion that BHIs may be mediated not only via nerval structures but also a humorally. It has to be mentioned that the brain is also a hormone and cytokineproducing organ. Particularly, the pituitary gland produces a number of substances that have either a direct or an indirect effect on heart functions. Hormones of the anterior lobe of the pituitary gland affect the hypothalamic-pituitary adrenal axis, and hormones from the posterior lobe affect the heart indirectly via the regulation of the blood volume.

Another shortcoming is that there may not be an interaction with the "heart" in general but with specific structures of the heart. These include the myocardium, the cardiac conduction system, the coronary arteries, cardiac venes, the pericardium, endocardium, and the autonomic innervation. There should be a discussion about which of these structures are predominantly affected in the various different $\mathrm{BH}$.
${ }^{1}$ Klinik Landstrasse, Messerli Institute, Vienna, Austria

${ }^{2}$ Programa de Estudos Pós-Graduado em Fonoaudiologia, Pontifícia Universidade Católica de São Paulo (PUC-SP), Departamento de Fonoaudiologia, Escola Paulista de Medicina/Universidade Federal de São Paulo (EPM/UNIFESP), São Paulo, Brasil

${ }^{3,4}$ Disciplina de Neurociência, Escola Paulista de Medicina/Universidade Federal de São Paulo, (EPM/UNIFESP), São Paulo, Brasil

Corresponding Author: Josef Finsterer, Klinik Landstrasse, Messerli Institute, Vienna, Austria, Phone: +43-1-71165-72085, e-mail: fifigs1@ yahoo.de

How to cite this article: Finsterer J, Fiorini AC, Scorza CA, Scorza FA. Brain-Heart Interactions are More Diverse than Anticipated. Indian J Crit Care Med 2020;24(10):1003-1004.

Source of support: Nil

Conflict of interest: None

Unaddressed in the review is that direct or indirect lesions of the vagal nerve may a have a strong impact on the autonomic innervation of the heart. ${ }^{6}$ Overstimulation of the vagal nerve may result from brain stem lesions or lesions in the extra-cerebral but intracranial course of the vagal nerve.

Unaddressed remain neurological patients who have implanted microelectrodes in their brain. Particularly, from stimulators used for deep brain stimulation of the thalamus, globus pallidus, or the subthalamic areas (tremor, Parkinson, dystonia, and epilepsy), it is known that they may affect the vagal nerve as a side-effect. ${ }^{7}$

Finally, the brain should not be only regarded as topically organized but also organized in network structures that interact with each other or with various other humoral or nerval systems in the body, a perspective that should be included in the discussion about BHIs.

In summary, the important issue of BHls needs to be more extensively discussed, and topics, such as SUDEP, SUDPAR, neuronal networks, endocrine cerebral functions, drugs affecting the brain, and cerebral microelectrodes, should be addressed. Widening the spectrum of BHIs is important, as the issue is still poorly understood and still not widely recognized.

\section{Author Contribution}

Josef Finsterer contributed in design, literature search, discussion, first draft, critical comments.

\section{REFERENCES}

1. Prasad Hrishi A, Ruby Lionel K, Prathapadas U. Head rules over the heart: cardiac manifestations of cerebral disorders. Indian J Crit Care Med 2019;23(7):329-335. DOI: 10.5005/jp-journals-10071-23208.

(0) The Author(s). 2020 Open Access This article is distributed under the terms of the Creative Commons Attribution 4.0 International License (https://creativecommons. org/licenses/by-nc/4.0/), which permits unrestricted use, distribution, and non-commercial reproduction in any medium, provided you give appropriate credit to the original author(s) and the source, provide a link to the Creative Commons license, and indicate if changes were made. The Creative Commons Public Domain Dedication waiver (http://creativecommons.org/publicdomain/zero/1.0/) applies to the data made available in this article, unless otherwise stated. 
2. Hering D, Trzebski A, Narkiewicz K. Recent advances in the pathophysiology of arterial hypertension: potential implications for clinical practice. Pol Arch Intern Med 2017;127:195-204. DOI: 10.20452/ pamw.3971.

3. Whitney R, Donner EJ. Risk factors for sudden unexpected death in epilepsy (SUDEP) and their mitigation. Curr Treat Options Neurol 2019;21(2):7. DOI: 10.1007/s11940-019-0547-4.

4. Jin L, Zhang Y, Wang XL, Zhang WJ, Liu YH, Jiang Z. Postictal apnea as an important mechanism for SUDEP: a near-SUDEP with continuous EEG-ECG-EMG recording. J Clin Neurosci 2017;43:130-132. DOI: 10.1016/j.jocn.2017.04.035.
5. Nejm MB, Andersen ML, Tufik S, Finsterer J, Scorza FA. Sudden death in Parkinson's disease: unjustifiably forgotten. Parkinsonism Relat Disord 2019;58:88-89. DOI: 10.1016/j.parkreldis.2018.08.012.

6. Leviter J, Wiznia DH. Carotid space mass proximal to vagus nerve causing asystole and syncope. Case Rep Neurol Med 2016;2016:9306784. DOI: 10.1155/2016/9306784.

7. Kanagaratnam L, Lee A, Whalley D, Figtree GA. Overcoming artifacts and fears: electrophysiology study and radiofrequency ablation in a Parkinsonian patient with supraventricular tachycardia and a brain neurostimulator. Pacing Clin Electrophysiol 2013;36(1):e1-e3. DOI: 10.1111/j.1540-8159.2011.03204.x. 